typeset using JPSJ.sty <ver.1.0b $>$

\title{
Competition between spin exchange and correlated hopping
}

\author{
Y. Saiga ${ }^{a}$, M. Imadab \\ ${ }^{a}$ Department of Physics, Tokyo Institute of Technology, 2-12-1 Oh-okayama, Meguro-ku, Tokyo 152-8551 \\ ${ }^{\mathrm{b}}$ Institute for Solid State Physics, University of Tokyo, Kashiwa-no-ha 5-1-5, Kashiwa 277-8581
}

(Received

)

\begin{abstract}
The ground-state phase diagram is numerically studied for an electronic model consisting of the spin exchange term $(J)$ and the correlated hopping term $\left(t_{3}\right.$ : the three-site term). This model has no single-particle hopping and the ratio of the two terms is controlled by a parameter $\alpha \equiv 4 t_{3} / J$. The case of $\alpha=1$ corresponds to complete suppression of single-particle hopping in the strongcoupling limit of the Hubbard model. In one dimension, phase separation takes place below a critical value $\alpha_{\mathrm{c}}=0.36-0.63$ which depends on the electron density. Spin gap opens in the whole region except the phase-separated one. For $\alpha \gtrsim 1.2$ and low hole densities, charge-density-wave correlations are the most dominant, whereas singlet-pairing correlations are the most dominant in the remaining region. The possibility of superconductivity in the two-dimensional case is also discussed, based on equal-time pairing correlations.
\end{abstract}

KEYWORDS: A. Superconductors; D. Phase transitions; D. Superconductivity

The discovery of high- $T_{\mathrm{c}}$ cuprates 1 has driven us the question whether the new microscopic theory for pairing mechanism is required. In the cuprates strong Coulomb repulsion may be essential, and various pairing mechanisms have been proposed under strong correlation. One of the common features in experiment is the extremely flat band around the momenta $(0, \pi)$ and $(\pi, 0)$ in units of the reciprocal lattice parameter, which has been observed in angle-resolved photoemission spectroscopy (ARPES).2) This feature is attributed to a strongly correlated effect. The band structure similar to the ARPES data has been numerically obtained for the Hubbard model with strong on-site Coulomb repulsion and the $t$ - $J$ modele in two dimensions.

If the flat band plays a crucial role in increasing the critical temperature, proper control of the single-particle process should lead tomore enhancement of superconducting correlations. 0 . Wumerical results for onedimensional (1D) electron systems show that flattening of the band near the Fermi points actually extends the parameter region where pairing correlations are the most dominant If single-particle hopping is completely absent as inessentials, other hopping mechanism is necessary for superconductivity. One of the candidates is the correlated hopping, which is often called the three-site term and is naturally derived in the strong-coupling expansion of the Hubbard model. (9) However, in one dimension, the correlated hopping is known to be suppress pairing correlations in the low-hole-density region 10 , 41 This is because the correlated hopping leads to a repulsion between holes. (1) In contrast, spin exchange interaction favors the attractive configuration of electrons. Therefore the correlated hopping and the spin exchange should compete in an electron-density region, and the ground-state phase diagram is nontrivial.

\footnotetext{
* E-mail address: saiga@stat.phys.titech.ac.jp
}

In this paper, we shall clarify the ground-state properties due to the competition between spin exchange and correlated hopping in one dimension. We also discuss realization of superconductivity under complete suppression of single-particle hopping in the $2 \mathrm{D}$ case. To this end, exact diagonalization is employed for finite systems.

In the Hilbert space without double occupancy of electrons, the strong-coupling expansion of the Hubbard model yields the following Hamiltonian up to the order $t^{2} / U$ ( $U$ being the on-site Coulomb repulsion):

$$
\begin{gathered}
\mathcal{H}=\mathcal{H}_{t}+\mathcal{H}_{J}+\mathcal{H}_{t 3}, \\
\mathcal{H}_{t}=-t \sum_{\langle i j\rangle \sigma}\left(\tilde{c}_{i \sigma}^{\dagger} \tilde{c}_{j \sigma}+\text { H.c. }\right), \\
\mathcal{H}_{J}=J \sum_{\langle i j\rangle}\left(\boldsymbol{S}_{i} \cdot \boldsymbol{S}_{j}-\frac{1}{4} n_{i} n_{j}\right), \\
\mathcal{H}_{t 3}=-t_{3} \sum_{\langle i j \ell\rangle \sigma}\left(\tilde{c}_{i \sigma}^{\dagger} n_{j,-\sigma} \tilde{c}_{\ell \sigma}\right. \\
\left.-\tilde{c}_{i \sigma}^{\dagger} \tilde{c}_{j,-\sigma}^{\dagger} \tilde{c}_{j \sigma} \tilde{c}_{\ell,-\sigma}+\text { H.c. }\right),
\end{gathered}
$$

where $\langle i j\rangle$ and $\langle i j \ell\rangle$ are nearest neighbors, $J=4 t^{2} / U$ and $t_{3}=\alpha J / 4$ with $\alpha=1$. The constrained fermion operator $\tilde{c}_{i \sigma}$ is given by $\tilde{c}_{i \sigma}=c_{i \sigma}\left(1-n_{i,-\sigma}\right)$. Here we consider the value of $\alpha$ as a continuous parameter. In one dimension, the ground-state phase diagram in the $J / t-n$ plane ( $n$ being the electron density) has been investigated for $\alpha=0,2$ ) and several values of $\alpha>0.10$ We refer to a model with $\mathcal{H}_{t}$ deleted, namely $\mathcal{H}_{J t 3} \equiv \mathcal{H}_{J}+\mathcal{H}_{t 3}$, as the $J-t_{3}$ model. We will complete a phase diagram in the $\alpha-n$ plane for the $J-t_{3}$ model. Originally, both $\mathcal{H}_{J}$ and $\mathcal{H}_{t 3}$ decay more rapidly than $\mathcal{H}_{t}$ when the hopping integral $t$ approaches zero. In considering possible mechanisms for superconductivity, however, it would be meaningful to deal with each term of eq. (1) individually away from the Hubbard model. 
It should be noted that in the $1 \mathrm{D} J-t_{3}$ model the set of all eigenvalues under the antiperiodic boundary condition is equivalent to that under the periodic one.

We first discuss the phase diagram in the $1 \mathrm{D}$ case. In order to estimate the boundary of phase separation, we calculate the compressibility given by

$\kappa=\frac{N}{N_{\mathrm{e}}^{2}} \cdot \frac{4}{E_{0}\left(N ; N_{\mathrm{e}}+2\right)+E_{0}\left(N ; N_{\mathrm{e}}-2\right)-2 E_{0}\left(N ; N_{\mathrm{e}}\right)}$,

where $E_{0}\left(N ; N_{\mathrm{e}}\right)$ denotes the ground-state energy with $N_{\mathrm{e}}$ electrons in $N$ sites. In Fig. 1 we show the values of $\alpha_{\mathrm{c}}$ at which $1 / \kappa$ crosses zero for $N=10-16$. The size dependence is small and this implies a reliable estimate of the thermodynamic results. The phase separation occurs for $\alpha<\alpha_{\mathrm{c}}$.

In the low-electron-density limit, one can estimate the phase boundary by solving two-electron problem.13) In the $J-t_{3}$ model, the phase separation disappears at $n \sim 0$ for $\alpha>\alpha_{\mathrm{c}}=2 \ln 2-1 \simeq 0.386$.10) This estimate would be exact if there are no bound states with more than two electrons. In the high-electron-density limit, on the other hand, one may estimate the boundary from balance between the gain in kinetic energy and the loss of exchange epergy due to insertion of two holes in a Heisenberg chain.12 Following ref. 12) we calculate the groundstate energy with $N-2$ electrons in $N$ sites relative to the Heisenberg energy in $N-2$ sites. This energy becomes negative for $\alpha>0.497,0.488,0.483$ and 0.480 for $N=10,12,14$ and 16 , respectively. We obtain $\alpha_{\mathrm{c}} \sim 0.47$ from extrapolation to the thermodynamic limit. This value is smaller than $\alpha_{\mathrm{c}} \sim 0.63$ obtained from the compressibility at the highest electron density $(n=0.875)$ in our calculation. Anyway the critical values at $n \sim 0$ and $n \sim 1$ are both smaller than $\alpha=1$, which implies that the $\alpha=1$ line is not interrupted by phase separation.

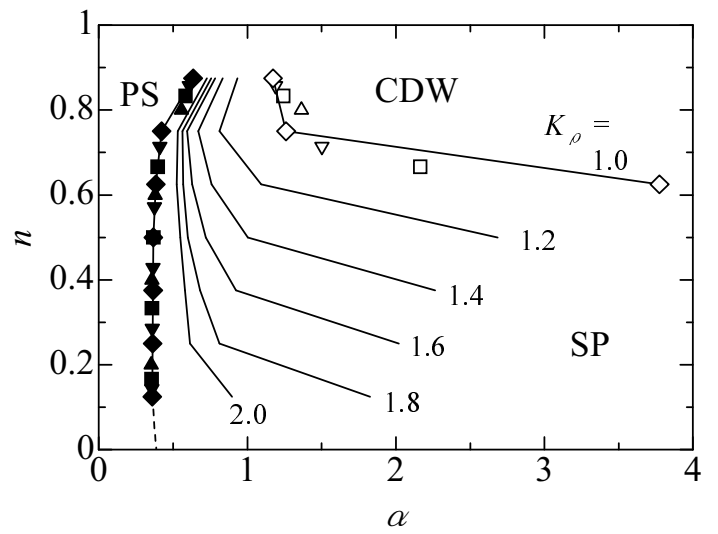

Fig. 1. Phase diagram for the ground state of the $1 \mathrm{D} J-t_{3}$ model. PS: phase-separated state; CDW: charge-density-wave state; SP: singlet-pairing state. Closed symbols and open ones show the phase-separated boundary and the contour of $K_{\rho}=1$, respectively, for $N=10$ (upward triangles), 12 (squares), 14 (downward triangles) and 16 (diamonds with a solid line). The contours of other values of $K_{\rho}$ for $N=16$ are shown by sqlid lines. The low-density limit of the phase-separated boundary 10 ) is shown by a broken line.

We next calculate the spin gap given by $E_{\mathrm{SG}}=$ $\lim _{N \rightarrow \infty} E_{\mathrm{GS}}\left(N ; N_{\mathrm{e}}=N n\right)$, where $E_{\mathrm{SG}}\left(N ; N_{\mathrm{e}}\right)=$ $E_{0}\left(N ; N_{\mathrm{e}} ; S_{\text {tot }}^{z}=1\right)-E_{0}\left(N ; N_{\mathrm{e}} ; S_{\text {tot }}^{z}=0\right)$. Here $S_{\text {tot }}^{z}$ denotes the $z$-component of total spin for the system. We estimate the magnitude of spin gap with use of the data of $N=9,12,15$ for $n=2 / 3, N=8,12,16$ for $n=0.5$, and $N=6,12,18$ for $n=1 / 3$. The following fitting function is used for extrapolation to $N \rightarrow \infty$ :10)

$$
\Gamma(N)=\Gamma(\infty)+A / N^{m}+B / N^{2 m},
$$

with $\Gamma=E_{\mathrm{SG}}$ and $m=1$. Figure 2 shows the extrapolated values of the spin gap as a function of $\alpha$. At any electron density, the presence of spin gap is clearly shown for the whole region of $\alpha>\alpha_{\mathrm{c}}$. In the inset of Fig. 2, both horizontal and vertical axes are scaled by $\alpha$. This figure makes the continuity to a case of only $\mathcal{H}_{t 3}$ (i.e., $1 / \alpha=0) 11$ clear. The spin gap scaled by $\alpha$ has a peak near $\alpha=1$ at any filling.

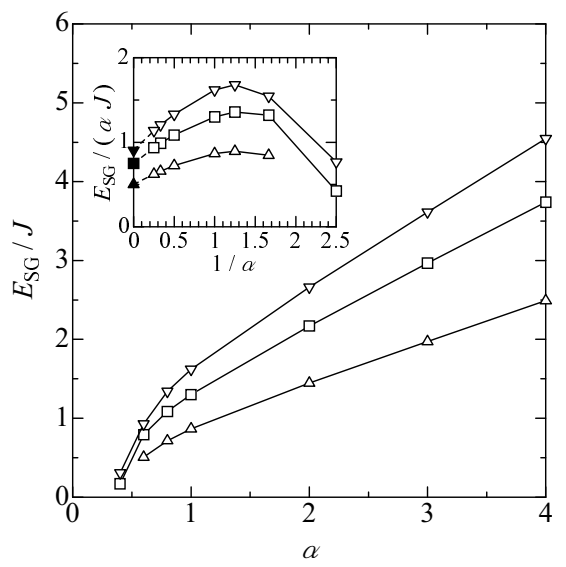

Fig. 2. Spin gap versus $\alpha$ for the $1 \mathrm{D} J-t_{3}$ model. Each value is extrapolated from the finite-size data. The values for $n=$ $2 / 3,1 / 2$ and $1 / 3$ are shown by upward triangles, squares and downward triangles, respectively. The inset shows the spin gap scaled by $\alpha$ as a function of $1 / \alpha$. The value of the analytic result for only the correlated hopping $(1 / \alpha=0) 11$ are indicated by closed symbols.

An accurate way to estimate a spin-gap region is the singlet-triplet level crossing method 14, 15. Nakamura et al. applied the method to the $1 \mathrm{D} t-J$ model, assuming that the low-energy behavior of the model is described by the effective Hamiltonian consisting the U(1) Gaussian model and the $\mathrm{SU}(2)$ sine-Gordon model.16) One can check the validity by seeing that $x_{r} \equiv\left(x_{\mathrm{ss}}+3 x_{\mathrm{st}}\right) / 4$ is close to $1 / 2$, where $x_{\mathrm{ss}}\left(x_{\mathrm{st}}\right)$ denotes the scaling dimension of the singlet (triplet) excitations. These excitations are calculated under the boundary condition twisted from the one for the ground state.16.

When the level crossing method is applied to the $J-t_{3}$ model, we should notice two points. One is the presence of correlated hopping, and the other is the absence of single-particle hopping. Here we assume that in the lowenergy region the model given by eq. (1) is described by the above effective Hamiltonian even for $t_{3}>0$. The validity is checked from the scaling dimension to be shown later. Meanwhile, the presence of single-particle hopping $t$ seems to be indispensable because it provides the 
finite gradient for linearization of the band dispersion. To avoid the ambiguity, we begin with the finite value of $t$, and then consider the limit of $t \rightarrow 0$.

Figure 3(a) shows the spin-gap boundary obtained by the level crossing method, in the $\alpha-t / J$ plane at $n=0.5$ and 0.875 in 16 sites. There is no spin gap in the region above the solid line [case $(\mathrm{A})$ ], while the remaining region should be the spin-gap region or the phase-separated one [case (B)]. Obviously the limit of $t \rightarrow 0$ belongs to the case (B) at both electron densities. The validity of the method is ensured in Fig. 3(b), which shows that the scaling dimension $x_{r}$ in the gapless region $(t / J \gtrsim 0.8)$ is very close to $1 / 2(0.502-0.504)$ for $N=16, n=0.5$ and $\alpha=1$. One may ask whether the limit of $t \rightarrow 0$ and the case of $t=0$ are connected smoothly. In Fig. 3(c) we show the magnitude of spin gap as a function of $t / J$. The figure informs us no singularity of the spin gap at $t / J=0$. This situation would hold also for other values of $\alpha$ and fillings unless phase separation occurs. Thus we conclude that the $J-t_{3}$ model has a spin gap in the whole region except the phase separated one.17)

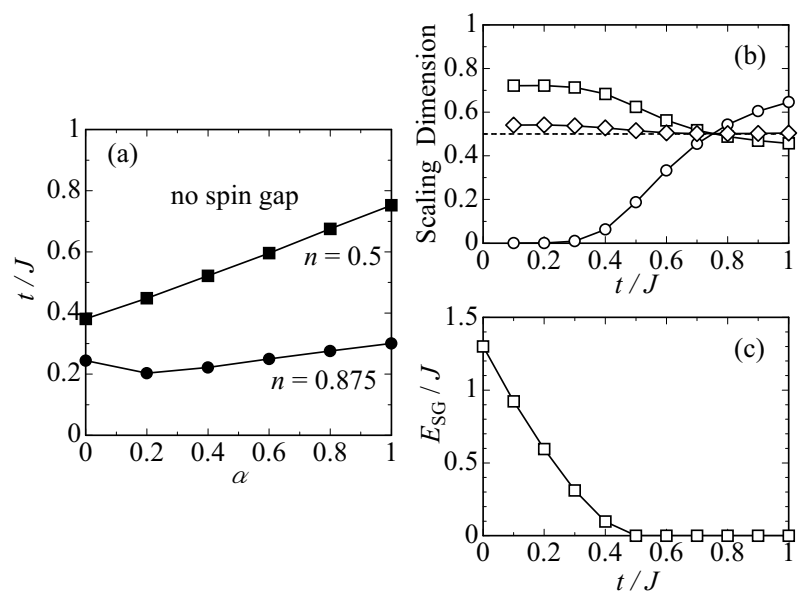

Fig. 3. (a) Spin-gap boundary in the $\alpha-t / J$ plane at $n=0.5$ and 0.875. (b) Scaling dimension of the lowest singlet excitation $\left(x_{\mathrm{SS}}\right)$ and that of the triplet excitation $\left(x_{\mathrm{st}}\right)$ for $N=16, n=0.5$ and $\alpha=1$. The quantities $x_{\mathrm{ss}}, x_{\mathrm{st}}$ and $x_{r} \equiv\left(x_{\mathrm{ss}}+3 x_{\mathrm{st}}\right) / 4$ are shown by circles, squares and diamonds, respectively. (c) Magnitude of spin gap as a function of $t / J$ for $n=0.5$ and $\alpha=1$. Each value is extrapolated from the finite-size data $(N=8,12,16)$.

Although the $J-t_{3}$ model has no single-particle hopping, the charge velocity is not zero but finite due to the correlated hopping.11] Thus we expect that charge degrees of freedom for the system behave as a TomonagaLuttinger (TL) liquid. To support this, we calculate the central charge $c$ which characterizes the universality class of a model. One can obtain $c$ by fitting the ground-state energy as eq. (6) with $\Gamma=E_{0} / N$ and $m=2$. Here the coefficient $A$ is given by $-\pi v_{\mathrm{c}} c / 6$, and the charge velocity $v_{\mathrm{c}}$ is extrapolated by eq. (6) with $\Gamma=v_{\mathrm{c}}$ and $m=2$. Note that $A$ does not include the spin velocity due to the presence of spin gap. With use of the data of $N=8,12$ and 16 with $n=0.5$, we find that $c$ is almost unity $(c \sim 0.999)$ in the region $\alpha=0.6-4.0$. This in- dicates that charge degrees of freedom are described by a TL liquid. For $\alpha \lesssim 0.4, c$ deviates largely from unity, reflecting the phase separation which is no longer a TL liquid.

In the region $c=1$, one can use the following universal formula about the correlation exponent $K_{\rho}$ :

$$
K_{\rho}=\pi \sqrt{\frac{D n^{2} \kappa}{2}},
$$

where the Drude weight $D$ is defined as

$$
D=\left.\frac{N}{2} \frac{\partial^{2} E_{0}(\phi)}{\partial \phi^{2}}\right|_{\phi=0} .
$$

Here $E_{0}(\phi)$ denotes the ground-state energy of the system under the twisted boundary condition $\left(c_{i+N, \sigma}=\right.$ $\left.\mathrm{e}^{-\mathrm{i} \phi} c_{i \sigma}\right)$. When a spin gap is present, singlet-pairing [charge-density-wave (CDW)] correlation functions depend on the distance $r$ between sites as $r^{-1 / K_{\rho}}\left[r^{-K_{\rho}}\right]$ for $r \gg 1$. If $K_{\rho}>1$, singlet-pairing correlations are the most dominant; otherwise CDW correlations are the most dominant. In Fig. 1 we show the contour lines of $K_{\rho}$ with 1.0-2.0.

Our result for the values of $K_{\rho}$ is supported by direct calculation of the equal-time pairing correlation function $C(r)=(1 / N) \sum_{i}\left\langle P_{i}^{\dagger} P_{i+r}\right\rangle$. Here the singlet pairing operator $P_{i}$ is given by $P_{i}=\left(c_{i \uparrow} c_{i+1, \downarrow}-c_{i \downarrow} c_{i+1, \uparrow}\right) / \sqrt{2}$, and $\langle\dot{\sim}\rangle$ denotes the expectation value in the ground state.18) Figures $4(\mathrm{a})$ and 4(b) show the distance dependence of $C(r)$ for various values of $\alpha$ with $n=0.75$ fixed and that for various fillings with $\alpha=\infty$ fixed, respectively. In any case, pairing correlations reach up to long distance for $K_{\rho}>1$. The sum $S=\sum_{r} C(r)$ provides the uniform (zero-momentum) component of the pairing structure factor. Figure 4(c) shows the result of $S$ as a function of $\alpha$ for various fillings. The values of $\alpha$ at which $S$ has a peak are found to correspond to the phase-separated boundary $\left(K_{\rho}=\infty\right)$ in Fig. 1 .

The phase diagram consists of three regions: the phase-separated region, the CDW one and the singletpairing one. Note that for $\alpha=\infty$ the CDW correlations are the most dominant in the range $n>2-\sqrt{2} \simeq$ 0.586.11) We emphasize that the pairing correlations are the most dominant irrespective of $n$ for $\alpha=1$ corresponding to complete suppression of single-particle hopping in the strong coupling limit of the Hubbard model.

How is the situation changed on a square lattice? We employ a $4 \times 4$ cluster with the periodic boundary condition in both $x$ and $y$ directions. To search indications of superconductivity, we calculate equal-time pairing correlations given by $C_{\mathrm{s}(\mathrm{d})}(\vec{r})=(1 / N) \sum_{\vec{i}}\left\langle\Delta_{ \pm}^{\dagger}(\vec{i}) \Delta_{ \pm}(\vec{i}+\right.$ $\vec{r})\rangle$ 18, 19 The pairing operator $\Delta_{ \pm}(\vec{i})$ is defined as $\Delta_{ \pm}(\vec{i})=c_{\vec{i} \uparrow}\left(c_{\vec{i}+\vec{\delta}_{x}, \downarrow}+c_{\vec{i}-\vec{\delta}_{x}, \downarrow} \pm c_{\vec{i}+\vec{\delta}_{y}, \downarrow} \pm c_{\vec{i}-\vec{\delta}_{y}, \downarrow}\right)$, where + $(-)$ corresponds to extended-s-wave $\left(\mathrm{d}_{x^{2}-y^{2}}\right.$-wave) symmetry, and the unit vector in $x(y)$ direction is represented by $\vec{\delta}_{x}\left(\vec{\delta}_{y}\right)$.20) In Fig. 5(a) we show the distance dependence of $C_{\mathrm{s}}(r)$ and $C_{\mathrm{d}}(r)$ with $r \equiv|\vec{r}|$ for $n=0.5$ and $\alpha=1$. The extended-s-wave pairing correlations are

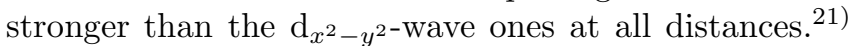
Figure 5(b) shows the extended-s-wave correlations for $n=0.5$ and various values of $\alpha$. The rapid decay at 


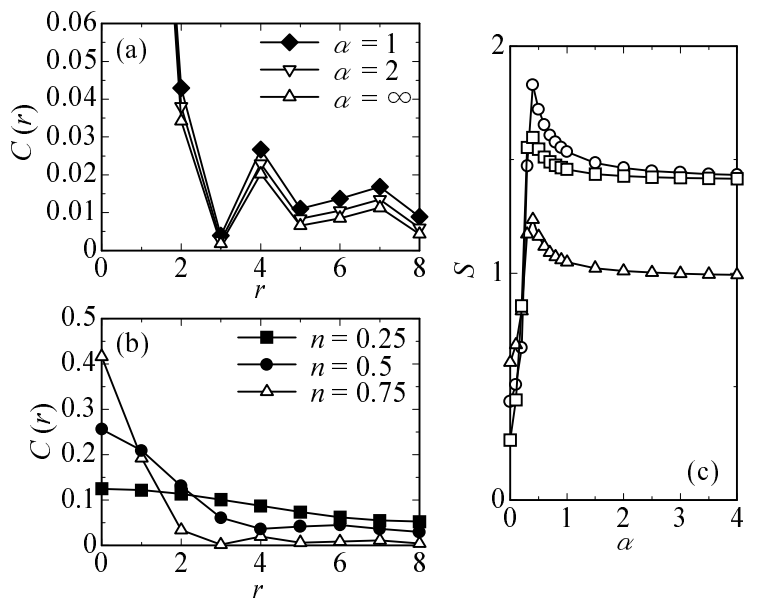

Fig. 4. (a) Equal-time pairing correlation function as a function of distance for the 1D $J-t_{3}$ model with $N=16, n=0.75$ and three different values of $\alpha$. (b) Same as (a) but with $\alpha=\infty$ and three different values of filling. In (a) and (b), cases indicated by closed symbols are within the singlet-pairing region $\left(1<K_{\rho}<\right.$ $\infty$ ) in Fig. 1. (c) Uniform component of the pairing structure factor as a function of $\alpha$ at $n=0.25$ (squares), 0.5 (circles) and 0.75 (upward triangles) in a 16-site cluster.

long distances for $\alpha=0.2$ reflects phase separation. The behavior for $\alpha=1$ and $\alpha=\infty$ (i.e., only the correlated hopping) is almost the same. This means that the pairing correlations are hardly affected by the spin exchange unless phase separation occurs. Finally we show the uniform component of the pairing structure factor $S_{\mathrm{s}(\mathrm{d})}=\sum_{\vec{r}} C_{\mathrm{s}(\mathrm{d})}(\vec{r})$ in Fig. 5(c). For $n=0.25$ and 0.5, we observe the abrupt increase in $S_{\mathrm{s}}$ as a function of $\alpha$. This implies a transition from phase separation to s-wave superconductivity. At fixed $\alpha(\gtrsim 0.4)$, as the electron density increases the extended-s-wave correlations become weak. Instead the $\mathrm{d}_{x^{2}-y^{2}}$-wave correlations become strong, but these do not excel the s-wave ones at least for $n \leq 0.75$.

In summary, we have found that the phase diagram of the $1 \mathrm{D} J-t_{3}$ model is composed of the three different regions. Most of the parameter space is occupied by the singlet-pairing region. Also in two dimensions, extendeds-wave superconducting states are likely to cover a wide range, although we have not confirmed whether the longrange order exists. A characteristic feature of the superconductivity discussed in this paper is to be caused by only the two-particle process. This mechanism is essentially different from the Bardeen-Cooper-Schrieffer (BCS) mechanism22 which is based on the single-particle process and an attractive potential between two electrons.

One of the authors (Y.S.) is grateful to M. Oshikawa, K. Okamoto and M. Kohno for valuable discussions. Y.S. is supported by JSPS Research Fellowships for Young Scientists.

[1] J. G. Bednorz, K. A. Müller, Z. Phys. B 64 (1986) 189.

[2] D. S. Dessau et al., Phys. Rev. Lett. 71 (1993) 2781.

[3] N. Bulut et al., Phys. Rev. B 50 (1994) 7215.
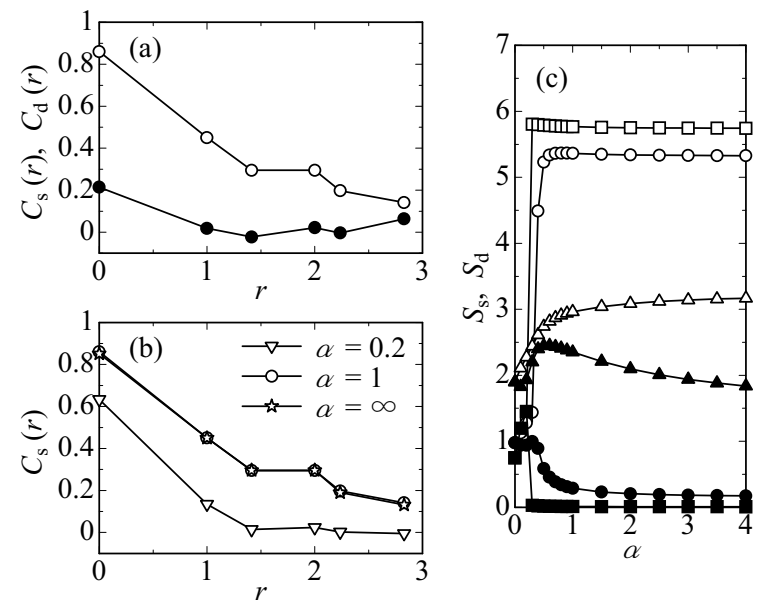

Fig. 5. (a) Equal-time pairing correlation function as a function of distance for the $2 \mathrm{D} J-t_{3}$ model with $N=4 \times 4, n=0.5$ and $\alpha=1$. Open (closed) symbols denote extended-s-wave $\left(\mathrm{d}_{x^{2}-y^{2-}}\right.$ wave) correlations throughout Fig. 5. (b) Extended-s-wave correlations with three different values of $\alpha$. Other parameters are the same as in (a). (c) Uniform component of the pairing structure factor as a function of $\alpha$ at $n=0.25$ (squares), 0.5 (circles) and 0.75 (upward triangles).

[4] E. Dagotto et al., Phys. Rev. Lett. 73 (1994) 728.

[5] H. Tsunetsugu, M. Imada, J. Phys. Soc. Jpn. 68 (1999) 3162.

[6] M. Imada, M. Kohno, Phys. Rev. Lett. 84 (2000) 143.

[7] M. Kohno, M. Imada, J. Phys. Soc. Jpn. 69 (2000) 25.

[8] A. B. Harris, R. V. Lange, Phys. Rev. 157 (1967) 295.

[9] J. E. Hirsch, Phys. Rev. Lett. 54 (1985) 1317.

[10] B. Ammon et al., Phys. Rev. B 52 (1995) 629.

[11] C. D. Batista et al., Phys. Rev. B 52 (1995) 6223.

[12] M. Ogata et al., Phys. Rev. Lett. 66 (1991) 2388.

[13] V. J. Emery et al., Phys. Rev. Lett. 64 (1990) 475.

[14] I. Affleck et al., J. Phys. A 22 (1989) 511.

[15] K. Okamoto, K. Nomura, Phys. Lett. A 169 (1992) 433.

[16] M. Nakamura et al., Phys. Rev. Lett. 79 (1997) 3214.

[17] This statement cannot be confirmed in the limit $n \rightarrow 1$.

[18] The ground state is calculated in the subspace where the total momentum is zero.

[19] Equal-time pairing correlations have been investigated for the 2D $t$ - $J$ model. For example, E. Dagotto, J. Riera, Phys. Rev. Lett. 70 (1993) 682.

[20] In this work we restrict hole (electron) pairs to nearest neighbors.

[21] It has been found by a variational Monte Carlo method that the three-site term stabilizes the extended-s-wave state in the low-density region, H. Yokoyama, M. Ogata, J. Phys. Soc. Jpn. 65 (1996) 3615.

[22] J. Bardeen et al., Phys. Rev. 108 (1957) 1175. 